\title{
Afferent Innervation Influences the Development of Dendritic Branches and Spines via Both Activity-Dependent and Non-Activity-Dependent Mechanisms
}

\author{
A. H. Kossel, ${ }^{1}$ C. V. Williams, ${ }^{1}$ M. Schweizer, ${ }^{3}$ and S. B. Kater ${ }^{2}$ \\ ${ }^{1}$ Department of Anatomy and Neurobiology, Colorado State University, Fort Collins, Colorado 80523, 2 Department of \\ Anatomy and Neurobiology, University of Utah School of Medicine, Salt Lake City, Utah 84132, and 3Zentrum für \\ Molekulare Neurobiologie, 20246 Hamburg, Germany
}

\begin{abstract}
The present investigation uses an in vitro co-culture system to study the role of afferent innervation in early development and differentiation of hippocampal neurons. Our experiments indicate that the formation of two key morphological features, dendritic branches and dendritic spines, is induced by afferent innervation. Hippocampal neurons develop multiple dendritic branches and spines only when extensively innervated by living axonal afferents. No morphological changes occurred when hippocampal neurons were plated on other cell surfaces such as fixed axons or astrocytes. Furthermore, afferents exerted their effect locally on individual dendrites that they contacted. When one portion of the dendritic arbor of a neuron was contacted by afferents and the other portion was not, morphological effects were restricted to the innervated dendrites. Innervation of some of the dendrites on a neuron did not produce global effects throughout the neuron. Afferent-induced dendritic
\end{abstract}

branching is independent of activity, since branch induction was unaffected by chronic application of TTX or glutamate receptor blockers. In contrast, the formation of dendritic spines is influenced by activity. The number of developing spines was reduced when TTX or a cocktail of three glutamate receptor blockers was applied. Blockade of individual AMPA, NMDA, or metabotropic glutamate receptors did not affect the number of spines. These results, taken together, demonstrate that afferents can have a prominent influence on the development of postsynaptic target cells via both activity-dependent and non-activity-dependent mechanisms, indicating the presence of multiple signals. Accordingly, this suggests an important interplay between pre- and postsynaptic elements early in development.

Key words: spines; dendrites; branches; glutamate receptors; activity; hippocampus; development
In the past, much attention has focused on axons as key elements in the formation of appropriate connections in the nervous system. Similarly, dendrites and their outgrowth and differentiation play a crucial role in the development of the connectivity of a neuron and ultimately determine its functional properties. Differentiated dendrites of neurons in the cortex and hippocampus are morphologically characterized by dendritic branches and dendritic spines. Both of these features are important determinants of the structure of a neuron and contribute to its integrative properties, its connectivity, and the changes occurring therein during synaptic plasticity (Miller et al., 1985; Koch and Zador, 1993; Spruston et al., 1995; Yuste and Denk, 1995). An important issue in neuronal development, therefore, is to determine how the formation of the dendritic tree is regulated and which intrinsic and extrinsic signals are involved in dendritic differentiation.

Evidence of a role for extrinsic factors in the development of dendrites and spines is derived from various experiments. Timelapse experiments have demonstrated that dendritic branches and

\footnotetext{
Received Dec. 19, 1996; revised May 19, 1997; accepted June 4, 1997.

This work was supported by a Feodor-Lynen Fellowship from the Alexander von Humboldt Foundation to A.H.K. and by National Institutes of Health Grant NS 24683 to S.B.K. We thank Dr. Bob Lee for providing some of the electron microscope work for this study, Kathy Charters for excellent technical support with the cultures, and A. Shibata and Drs. Peter Guthrie and Tom Parks for comments on this manuscript.

Correspondence should be sent to Dr. Albrecht H. Kossel at his present address: Max-Planck-Institute for Psychiatry, Am Klopferspitz 18A, 82152 Muenchen-Martinsried/Germany.

Copyright (C) 1997 Society for Neuroscience $0270-6474 / 97 / 176314-11 \$ 05.00 / 0$
}

spines are very dynamic, with branches and spines continuously being added and removed throughout development (Dailey and Smith, 1996; Ziv and Smith, 1996). One interpretation of this dynamic behavior is that dendrites may be able to read and to be influenced by extrinsic signals. Thus, interactions of dendrites with their environment may allow them to adapt and actively contribute to establishing connectivity with the surrounding environment (Dailey and Smith, 1996).

Afferent innervation seems to play an important role in the development of postsynaptic neurons during all stages of development. Denervation studies have shown that presynaptic innervation has important effects on the formation of dendrites and spines as well as on their maintenance (Hamori, 1973; Parnavelas et al., 1974; Frotscher et al., 1977, 1981; Frotscher, 1983; Anderson and Flumerfelt, 1984; Deitch and Rubel, 1984; Zafirov et al., 1994). Evidence for a role of afferents in the development of postsynaptic dendritic architecture also arises from studies focusing on plasticity of the nervous system during development. Structural changes occurring during remodeling of presynaptic axon terminals have long been known to be essential for refinement of connections during development (Shatz, 1990; Dan et al., 1995). Studies have only recently identified similar structural changes in postsynaptic dendritic architecture. Dendritic architecture is significantly altered at the borders between segregated afferent fibers in the visual system. The asymmetric dendritic architecture suggests that either outgrowth or stabilization of dendrites and dendritic branches is being selectively altered in these cells. Furthermore, activity is known to be involved in these 
postsynaptic alterations in a manner similar to known roles for presynaptic sites (Katz and Constantine-Paton, 1988; Katz et al., 1989; Kossel et al., 1995). While latter evidence is derived mostly from in vivo experiments, in vitro experiments also have shown an interplay of afferents and activity in maturation and differentiation of dendrites (e.g., cerebellar neurons) (Baptista et al., 1994). Thus, activity seems to play a fundamental role in the development of neuronal connections via both presynaptic changes and alteration in postsynaptic dendritic architecture.

The present study focuses on the potential role of afferent innervation in the early differentiation of hippocampal neurons. Hippocampal neurons are a well studied model for plastic changes influenced by afferents and their neuronal activity. A unique culture system in which hippocampal neurons were grown together with explants of entorhinal cortex enabled us to investigate mechanisms by which afferents might alter the development of dendritic structure. We show that both activity-dependent and non-activity-dependent factors influence dendritic differentiation. Furthermore, we established that individual innervated dendrites can be induced to differentiate while uninnervated parts of the same dendritic tree remain undifferentiated. Together with previous results, our findings strongly suggest a continuous interplay between presynaptic innervation and postsynaptic differentiation from early on.

\section{MATERIALS AND METHODS}

Cultures. Explants of entorhinal cortex were prepared from embryonic day 18 (E18) rats (Lewis). Entorhinal cortex was removed from the cortical hemispheres according to Mattson et al. (1988). The tissue was transferred into dishes with MEM $+10 \%$ FBS (Life Technologies, Bethesda, MD; Hyclone, Logan, UT) and subsequently cut into $\sim 1 \mathrm{~mm}$ pieces. Between four and eight of these tissue pieces were plated in dishes with glass coverslips coated with poly-L-lysine (Sigma, St. Louis, MO). Coverslips were subdivided into two areas. Only in one half of the coverslip were explants plated to extend an axonal net. Explants were allowed to grow for up to 2 weeks, until they had developed a rich axonal network on one half of the coverslip. The medium was then changed to $\mathrm{N} 2.1$, and frozen hippocampal neurons (E18) were plated into the dishes at a density of $2000-4000 \mathrm{cells} / \mathrm{cm}^{2}$ as described by Mattson et al. (1988) At this low cell density, most neurons were separated by several dendritic field diameters, allowing only few interactions. This enabled us to study hippocampal neurons in one area of the dish isolated from each other while neurons in the other area of the same dish were growing on the axonal net contacted by a high density of afferents. Neurons were grown for $3 \mathrm{~d}$, and then glia beds were added to the cultures (Banker and Cowan, 1979). This method helped to condition the medium and to keep the neurons alive. Neurons were grown for up to $14 \mathrm{~d}$. Cultures were also kept without glia beds for $14 \mathrm{~d}$ to ensure that the conditioned medium did not have any effects on the morphological parameters studied in cells growing on the axonal net.

Pharmacology. All substances used were kept as stock solutions that were diluted to the final concentration using N2.1 medium. The following concentrations were used: TTX (Sigma) $1 \mu \mathrm{M}$, DNQX (Sigma; RBI, Natick, MA) $20 \mu \mathrm{M}$, APV (AP-5) (RBI) $100 \mu \mathrm{M}$, and ( \pm )-MCPG (Tocris Cookson) $250 \mu \mathrm{M}$. Blockers were added to cultures at day 5 , before any obvious effects of the afferent innervation could be observed on the cells. To maintain a continuously high level of activity of different drugs, medium was exchanged every day in dishes containing blockers as well as in control dishes with regular medium. Cultures were used for analysis between days 12 and 14

Labeling and analysis. For labeling, cultures were first transferred into observation medium containing DMEM-HEPES (Life Technologies) and then to a heated microscope stage. For staining we used DiI (Molecular Probes, Eugene, OR) dissolved in cod liver oil (1 mg in $200 \mu \mathrm{l})$ (Papa and Segal, 1996). The mixture was sonicated for $5 \mathrm{~min}$ to break down DiI crystals and to dissolve fluorescent dye in the oil. The oil-DiI mixture was put into patch electrodes and applied in the form of oil droplets to the soma of a neuron by using a picospritzer (General Valve, Fairfield, NJ). Cells were allowed to stain for 10-60 min, before the dish was fixed for $10 \mathrm{~min}$ in $4 \%$ paraformaldehyde in PBS. In a number of cases, in addition to hippocampal neurons, neurons and glia originating from the entorhinal cortex were found to grow on the axonal net in the immediate area surrounding the explant. We therefore avoided staining of any cells in an area close to the explant. Images were taken using a CCD camera (Photometrics) and a fluorescent illumination-equipped microscope (Nikon). Images for analysis of dendritic parameters were taken with a $40 \times$ objective and stored on computer; data were analyzed later using NIH-image software. The number of primary dendrites and dendritic branch points and the lengths of dendrites were subsequently determined. To determine the number of branch points, only dendritic extensions longer than $10 \mu \mathrm{m}$ were considered and counted as dendritic side branches. For analysis of spines, images were taken using a $100 \times$ objective. Spines were counted along several dendritic sections of different individual dendrites per cell. Most spines extended within the $x / y$ plane; however, pictures were also taken from focal planes above and below to visualize and count spines extending in the $z$ direction. Spines and branches were counted in a nonblind fashion; however, for each experiment, control cultures that were grown in parallel under the same conditions were measured for comparison. Quantitative data are presented as means + SEM.

Growth of afferents in stripes. We developed a method to grow entorhinal afferents along a nonadhesive substrate polyA [poly(2-hydroxy-ethyl methacrylate)] (Sigma) to guarantee sharp borders of afferents within the dish. PolyA $(50 \%)$ dissolved in ethanol was applied onto glass coverslips in three to four parallel stripes by using a pipette tip. After they dried, explants were plated as described above. After 2 weeks the axonal carpet had covered the polyA free parts of the dish but had not grown on the nonadhesive substrate, resulting in sharp borders of afferents. PolyA nonadhesive stripes were then peeled off the dish with a forceps. Subsequently, plated neurons grew on top of the axonal net as well as on the now exposed poly-L-lysine lanes. Neurons grew readily in these uncovered lanes, demonstrating that the nonadhesive substrate had been removed completely from the glass surface and the poly-L-lysine substrate was still present to allow neurons to grow. A substantial number of neurons grew close enough to the borders of afferents that one part of their dendrites contacted the afferents and the other part grew on the polylysine surface. Cells were stained and analyzed after $10-11 \mathrm{~d}$ of growth. A shorter time period was chosen in these experiments to induce branching but also to minimize the risk of axonal outgrowth across the borders. The only dishes that were used were those in which the borders formed by the axonal carpet remained sharp and distinct, without significant subsequent outgrowth of axons onto the now uncovered polylysine side.

Immunohistochemistry. Cells were fixed in $2 \%$ paraformaldehyde for 30 min and subsequently washed in PBS containing 10\% serum. Cells were incubated overnight in the primary antibody against synaptophysin (Chemicon, Temecula, CA), washed several times in PBS, and incubated for $1 \mathrm{hr}$ in a rhodamine-labeled secondary antibody (Vector Laboratories, Burlingame, CA).

Fixation for electron microscopy. Tissue culture medium was removed from the dishes, and cells were fixed in $3 \%$ glutaraldehyde in $0.1 \mathrm{M}$ phosphate buffer (PB), pH 7.2. After $1 \mathrm{hr}$ fixation, glutaraldehyde was removed, and cells were washed twice in $\mathrm{PB}$. A solution of $1 \% \mathrm{OsO}_{4}$ in PB was added to the dish for $1 \mathrm{hr}$, followed by dehydration in a graded series of ethanol up to $100 \%$. The cells were infiltrated and embedded in thin layers of Epon-resin mixture. Appropriate sections of cells were sectioned with an ultramicrotome (Sorvall MT-2), stained with uranyl acetate and lead citrate, and examined in a transmission electron microscope (Jeol 2000) at $100 \mathrm{kV}$.

\section{RESULTS}

Dissociated hippocampal neurons growing in low density cultures can be classified into three types of cells (Banker and Cowan, 1979): (1) neurons having a more or less symmetrical dendritic field, (2) neurons with bipolar morphology, and (3) neurons dominated by one prominent dendrite. Therefore, neurons derived from whole hippocampi at E18 contain a mix of various cell types. Because we were interested in general mechanisms of branch and spine formation of hippocampal neurons, we distinguished only between pyramidal and nonpyramidal-looking cells. Potential GABAergic neurons, which can be identified by certain characteristics of their dendrites such as length or thickness, were not considered (Benson et al., 1994). 


\section{Afferents enhance formation of dendritic branches of hippocampal neurons}

Hippocampal cells plated on top of a dense carpet of entorhinal axons readily extended axons and dendrites, indicating that the entorhinal axons provided a growth-promoting substrate for hippocampal neurons. Entorhinal axons formed such a dense network of axons that single fibers could hardly be resolved. From the onset of their outgrowth, dendrites of hippocampal neurons were in contact with and embedded in numerous axons, giving the potential of forming actual synaptic contacts (Fig. $1 A$ ). Single hippocampal neurons plated directly on polylysine, not in contact with the explant, were contacted by only a few axons from neighboring cells (Fig. $1 B$ ). To determine whether synaptic contacts were formed between hippocampal neurons and the axonal bed, cultures were stained with antibodies for the presynaptic marker synaptophysin. As shown in Figure $1 C$, synaptophysin staining revealed a distinctive punctate labeling around the cell body and along the dendrites of the neurons. Parts of the axons not in contact with a postsynaptic hippocampal neuron did not show any label. This demonstrates that hippocampal neurons plated on top of the entorhinal axons formed synapses and did induce a local expression of synaptophysin on the axonal bed: the expression remained restricted to that part of the axons that contacted postsynaptic target cells and formed synapses. The presence of autaptic synapses and synapses with neighboring neurons could be excluded, because only a small number of contact points between these axons and the dendritic tree existed (6-10 per whole dendritic tree). The presence of synapses with the entorhinal axons was additionally confirmed by electron microscopy. Numerous synaptic contacts were found along the dendrites. The micrograph in Figure $1 D$ shows examples of synapses between presynaptic terminals of entorhinal axons and adjacent postsynaptic thickenings on the postsynaptic dendritic site. Although hippocampal neurons were plated on top of the entorhinal axons well after most of the axons had already grown out, hippocampal neurons were able to form many synapses with the underlying axonal net.

To study the difference between cells growing on and off the explants, cells were grown for different periods of time and subsequently labeled with the fluorescent marker DiI to visualize their morphology and quantitatively determine their outgrowth. Neurons extended a thin, long, and often branched axon and many shorter, thick minor processes that developed later into unbranched dendrites. During the first $5 \mathrm{~d}$ of growth, both the neurons grown on polylysine and those in contact with entorhinal axons developed similarly. Dendritic length in both groups of neurons did not differ significantly (Mann-Whitney $U$ test; $p>$ 0.05 from each other). Furthermore, analysis of the number of dendritic branch points showed that neurons in both groups developed very few dendritic branches during the first $5 \mathrm{~d}$ of outgrowth (on polylysine: $2 \pm 0.15, n=28$; on axons: $3 \pm 0.8, n=$ $25)$, with the number of branch points again not being significantly different from each other in both groups of cells (MannWhitney $U$ test; $p>0.05$ ).

By day 6/7 in culture, however, a dramatic difference in the morphology of cells growing on polylysine or in contact with the entorhinal axons started to emerge (Fig. 2). Neurons in contact with the axonal net extended many dendritic side branches. After $14 \mathrm{~d}$ in culture, nearly all such neurons had developed a very complex dendritic tree with multiple secondary and tertiary dendrites, with an increase of an average of eight times more branches than at day 5 (Fig. $2 C$ ). In contrast, neurons growing on polylysine only tripled the number of side branches in the period from day 6 until day $14(n=48$; Mann-Whitney $U$ test; $p<0.05$ ). With only seven branch points on average, the number of dendritic branches in isolated neurons was dramatically different from cells grown on the axonal net for $14 \mathrm{~d}$ (on the net: $24 \pm 1.4, n=47$; isolated: $7 \pm$ $0.6, n=48$; Mann-Whitney $U$ test; $p<0.001$ ). This difference in dendritic branching pattern was observed in all cell types and pyramidal and nonpyramidal cells.

\section{Is activity involved in dendritic branching?}

Afferent activity has been implicated as an important factor in dendritic architecture. As shown above, neurons growing on the entorhinal axons were able to form synapses with the axonal net and were therefore subject to massive afferent innervation. Single neurons growing only on polylysine were interconnected by axons from neighboring cells; however, they lacked this massive innervation. Our hypothesis that afferents induce formation of branches as a consequence of providing spontaneous input to the innervated hippocampal neurons was tested by blocking spontaneous activity with TTX. TTX $(1 \mu \mathrm{M})$ was added to the culture medium starting at day 5 , shortly before an increase in branching occurred, and was present in the medium until day 14 when most of the branches were formed. To our surprise, chronic blockade of activity with TTX as shown in Figure 3 did not change the number of dendritic branches when compared with control cultures grown in parallel for the same period of time (MannWhitney $U$ test; $p>0.05)$.

\section{Is the activation of glutamate receptors involved in dendritic branching?}

Because spontaneous transmitter release can occur in the presence of TTX and therefore locally depolarize the postsynaptic site, we tested whether activation of glutamate receptors is involved in promoting branching of dendrites. We tested the effect of the AMPA receptor blocker DNQX, the NMDA receptor blocker AP-5, and the metabotropic receptor blocker MCPG, which inhibits unspecifically all different types of metabotropic receptors. Dendritic branching was unaffected by blockade of either the AMPA receptor by $25 \mu \mathrm{M}$ DNQX, the NMDA receptor by $100 \mu \mathrm{M}$ AP-5, or metabotropic glutamate receptors by 250 $\mu \mathrm{M}$ MCPG. In each of these experiments the number of dendritic branches was not changed significantly compared with control cultures grown in parallel (Fig. 3) (Mann-Whitney $U$ test; $p>$ 0.05 for all three groups). Also the combination of all three blockers did not affect dendritic branching $(p>0.05)$. These results suggest that activity and release of glutamate are not necessary for the initiation of dendritic branching. Entorhinal cortical axons therefore seem to provide another important signal that increases dendritic branching.

\section{Do other cell surfaces promote dendritic branching?}

Because contact with afferents was able to increase formation of dendritic branches in hippocampal neurons, we tested whether other cell surfaces that dendrites usually encounter in the intact brain might also induce branching. Neurons were plated on astrocytes derived from E18 or postnatal day 1 animals. Neurons grown on astrocytes for $14 \mathrm{~d}$ developed few branches $(4 \pm 0.7$; $n=18)$ comparable to control cells on polylysine $(7 \pm 0.57 ; n=$ 48) (Fig. 4). This suggests the presence of a specific signal on the afferents. To test for a possible substrate effect, entorhinal axonal nets were fixed in $2 \%$ paraformaldehyde for $5 \mathrm{~min}$ and thoroughly 

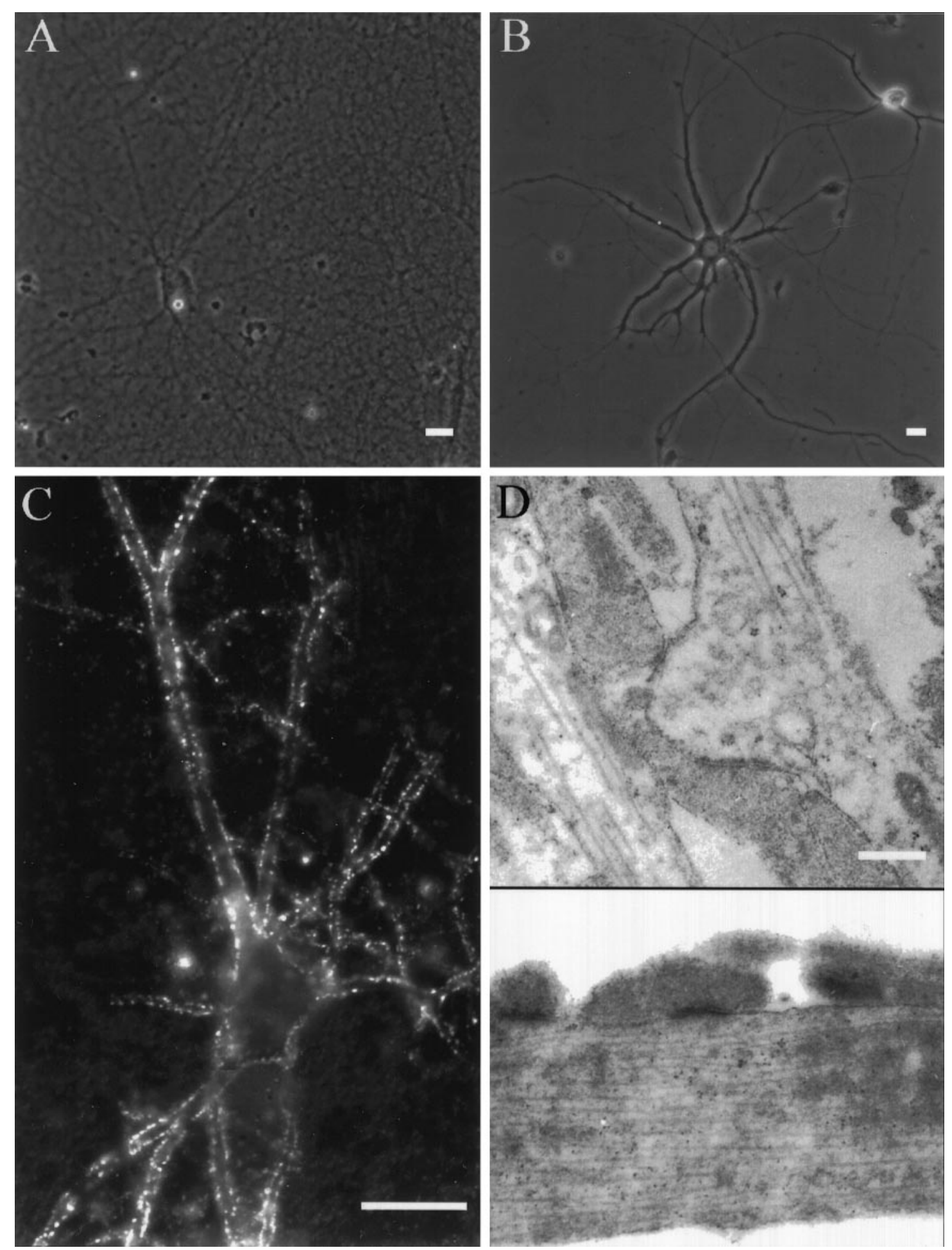

Figure 1. Innervation of neurons plated on a network of entorhinal axons and on polylysine. $A$, Hippocampal neurons grown on a dense net of entorhinal axons are embedded in an extensive meshwork of entorhinal axons, whereas neurons grown on polylysine $(B)$ are contacted by only a few axons from neighboring neurons at that cell density. $C$, Immunofluorescent staining with an antibody against synaptophysin shows punctated staining along the dendrites of a hippocampal pyramidal neuron growing on the axonal net for $14 \mathrm{~d}$, thus demonstrating the formation of synapses between the entorhinal axonal net and hippocampal neurons. The antibody, a marker for presynaptic terminals, stained only parts of the axons contacting the postsynaptic neuron. $D$, Electron micrograph showing the presence of numerous synapses between hippocampal neurons and the axonal net. Scale bars: $A-C, 25 \mu \mathrm{m} ; D, 0.5 \mu \mathrm{m}$.

washed in PBS and medium. Hippocampal neurons were then plated on top of these fixed axons. Neurons grown for $14 \mathrm{~d}$ on fixed axons were indistinguishable in their morphology from neurons grown on polylysine. Hippocampal neurons extended dendrites on this substrate. With only five branches on average, the neurons did not develop significantly more branches than neurons grown on polylysine (Fig. 4) (Mann-Whitney $U$ test; $p>$ $0.05)$. As shown in Figure 4 , the quantitative comparison of 

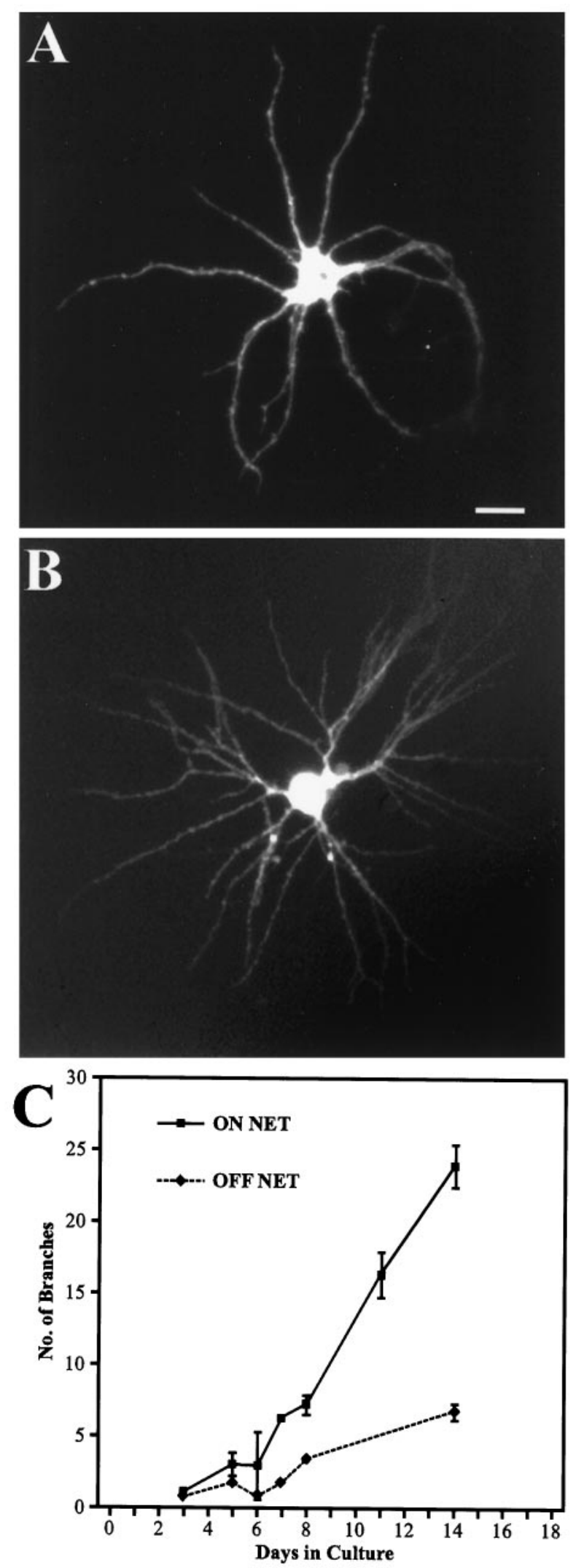

Figure 2. Development of dendritic branching dependent on the presence of innervation. Micrograph of hippocampal neurons growing off $(A)$ and on $(B)$ a net of entorhinal axons. Neurons growing on polylysine developed basal dendrites with no or only few branches $(A)$, whereas neurons growing in contact with the entorhinal axons have a highly branching on astrocytes, axons, and fixed axons indicates that living axons are required to induce branching.

\section{Can dendritic branching be induced locally?}

Afferents are able to induce the formation of dendritic branches in hippocampal neurons, suggesting the presence of a specific signal. This signal may act on neurons in two different ways. First, it may act locally on the dendrites it contacts and induce formation of individual branches. Second, contact with the entorhinal afferent net may provide an indirect signal to the neuron as a whole, activating an intrinsic program for dendritic branching.

To test the hypothesis that afferents provide a local signal, a unique culture system was designed in which some dendrites of a neuron were allowed to grow on the afferent net while other dendrites from the same neuron grew on polylysine. Distinct borders of afferents were achieved by growing entorhinal axons along a nonadhesive substrate (Fig. 5B) (for details, see Materials and Methods). As shown in Figure $5 B-D$, the afferents formed a clear border that remained distinct even after the nonadhesive substrate was removed. Dendrites growing into the axonal net and those growing in the opposite direction on polylysine were clearly distinguishable. Only dendrites extending into the afferent net developed a branched morphology (Fig. 5D,E). Dendrites of the same cell growing on the polylysine side remained unbranched. The obvious difference was also confirmed by a quantitative analysis of the border cells (Fig. 5F). Dendrites that touched the net developed an average of nine branch points per cell, about half of the branch points that were developed by neurons growing entirely on the net. The number of branch points for dendrites growing on polylysine on average was one. This significant difference between touching and nontouching dendrites was observed for basal dendrites from both pyramidal and nonpyramidal neurons as well as for apical dendrites of pyramidal neurons (Fig. 5D) (Mann-Whitney $U$ test; $p<0.001$ for apical and basal dendrites). These experiments strongly suggest that afferents induce emergence of dendritic branches by providing a local signal to the dendrites.

\section{Formation of dendritic spines in hippocampal neurons}

Dendritic spines are a distinct morphological feature of postsynaptic differentiation in hippocampal neurons. Dendritic spines emerge as characteristically short protrusions from the dendritic shaft at the sites of presynaptic glutamatergic synapses. As shown above, hippocampal neurons grown on entorhinal afferents form abundant synapses with the underlying axonal net. To study the morphological differentiation of spines at the postsynaptic site, neurons grown on the entorhinal net were visualized by fluorescent labeling with DiI. Dendrites of hippocampal neurons grown for 12-14 d were covered with many short protrusions having the characteristic shape of dendritic spines (Fig. 6A-C). Electron micrographs further confirmed the presence of spines on these neurons. They also showed spiny protrusions extending from dendrites grown on top of the axonal net that had the typical ultrastructure of spines, with a thin neck, a head, and a synaptic contact at the end

$\leftarrow$

differentiated dendritic tree with numerous branches $(B)$. $C$, Time course of development of dendritic branches for neurons growing on and off the axonal net. Neurons growing in contact with the axons started to develop highly branched dendritic trees after $6 \mathrm{~d}$ in vitro. After $14 \mathrm{~d}$ in culture these neurons had formed on average four times more branches than neurons growing off the net. Scale bar (shown in $A$ ): $25 \mu \mathrm{m}$. Error bars represent SEM. 


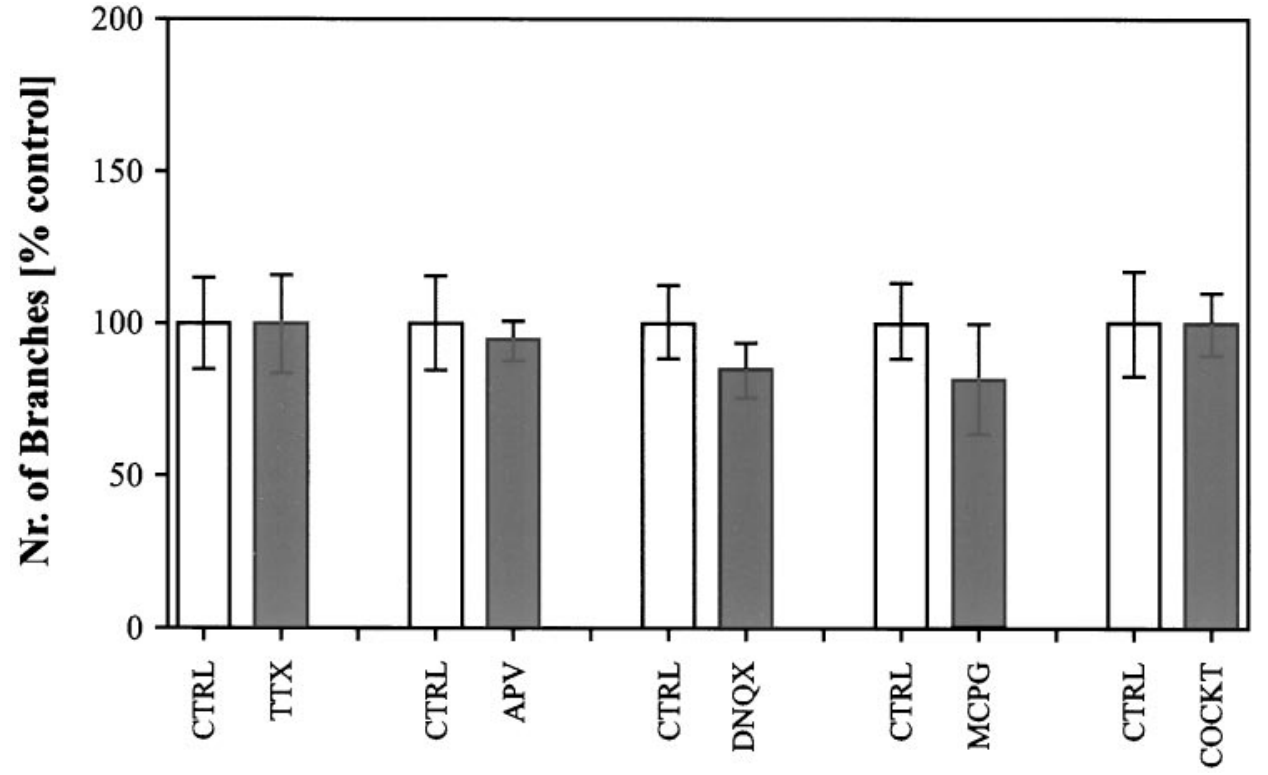

Figure 3. Role of activity and glutamate receptors in dendritic branching. The number of dendritic branches was not affected by chronic blockade of spontaneous activity with TTX $(n=48)$. Also, the chronic blockade of different glutamate receptors (AMPA, NMDA, metabotropic) by DNQX $(n=32)$, AP-5 $(n=27)$, and MCPG $(n=$ $15)$ alone or combined in a cocktail $(n=32)$ did not affect the branching. The average number of branches in treated cultures is shown as percentage of controls. Controls were performed in parallel to each experimental group to allow comparison (average number of branches in controls $=16.1 \pm$ $1.3 ; n=56$ ). Error bars represent SEM.
(Fig. 6D). Spine-like extensions from dendritic shafts without synapses were not observed. This strongly suggests that most of the protrusions seen by fluorescent label in the light microscope were indeed spines. These spiny protrusions were not present during the first $5 \mathrm{~d}$ but gradually started to develop thereafter. After $14 \mathrm{~d}$, neurons had developed an average of 13 spines per $100 \mu \mathrm{m}$ dendritic length ( $n=101$ cells).

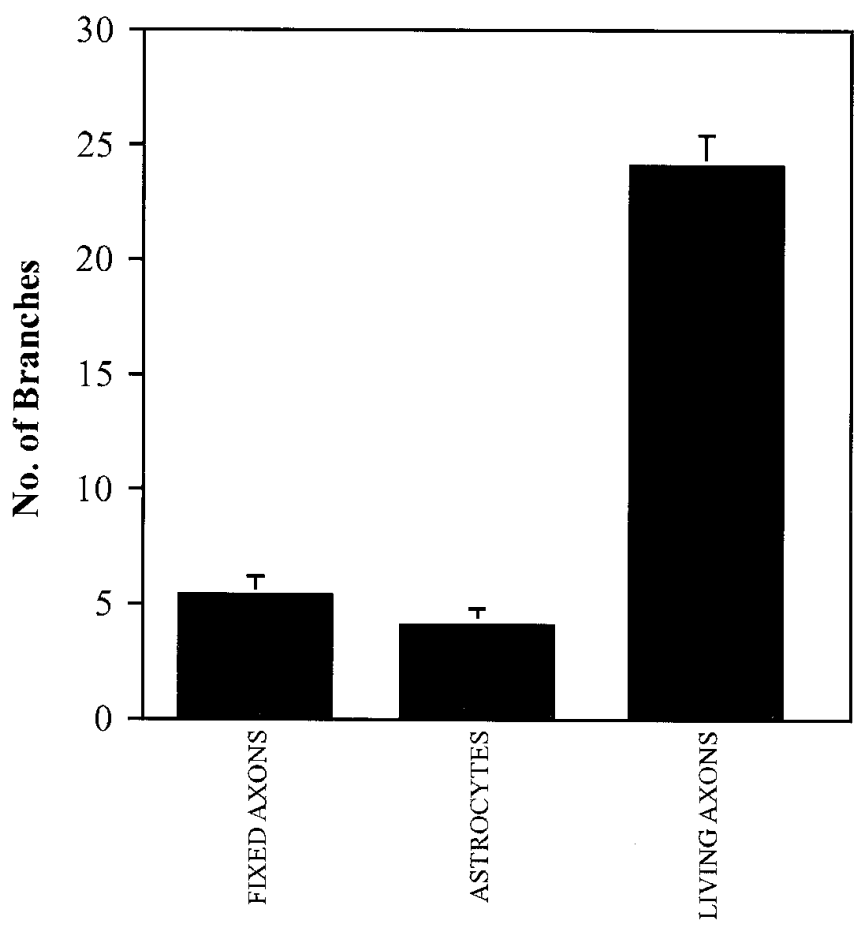

Figure 4. Role and specificity of different cell surfaces on dendritic branching. Neurons were grown on a fixed carpet of axons $(n=18)$ and a monolayer of living cortical astrocytes $(n=18)$ for $14 \mathrm{~d}$. On both surfaces hippocampal neurons remain unbranched. Only neurons contacting living axons developed stable dendritic branches $(n=48)$. The average number of branches is depicted. Error bars represent SEM.

\section{Is activity necessary for the development of spines?}

Many studies have shown that neuronal activity can influence shape and number of existing spines on a neuron (Horner, 1993; for review, see Harris and Kater, 1994). Because spines develop at the site of glutamatergic synapses, it is feasible that activity may exert its action through release of glutamate at the site of synaptic contact, depolarization, and influx of calcium at the postsynaptic site. This cascade of events may ultimately be involved in the alteration of the dendritic cytoskeleton; therefore, we tested the hypothesis that spontaneous activity from the entorhinal explants may be involved in the formation of spines in our culture system. We grew cultures under the chronic application of TTX $(1 \mu \mathrm{M})$ starting at day 5 , before the first obvious influence of afferent innervation on the morphology of the neuron could be detected. Neurons were grown in the presence of TTX until day 12-14, when a considerable number of spines usually developed. Results show that under chronic blockage of spontaneous activity by TTX, the number of spines was reduced by $50 \%$ compared with the control group (Fig. 7) ( $p<0.05$; Mann-Whitney $U$ test). The number of spines under TTX treatment was expressed relative to those in untreated control neurons. These were grown in parallel for the same time and consisted of the same batch of explants and neurons.

\section{Is activation of glutamate receptors necessary for the development of spines?}

To test further whether afferent activity exerts its effects through activation of glutamate receptors, cultures were grown under the chronic blockade of different glutamate receptor blockers. We tested the involvement of three different glutamate receptor types that have been shown to be involved in synaptic transmission between hippocampal neurons. The presence of the inhibitors for the AMPA receptor (25 $\mu \mathrm{M}$ DNQX), the NMDA receptor (100 $\mu \mathrm{M}$ AP-5), or the metabotropic glutamate receptor $(250 \mu \mathrm{m}$ MCPG) did not significantly affect the number of spines compared with their control groups (Fig. 7). We additionally tested for a combinatorial effect of all three different receptor blockers on the number of spines. Surprisingly, in cultures treated with a cocktail of all three blockers the number of spines was signifi- 

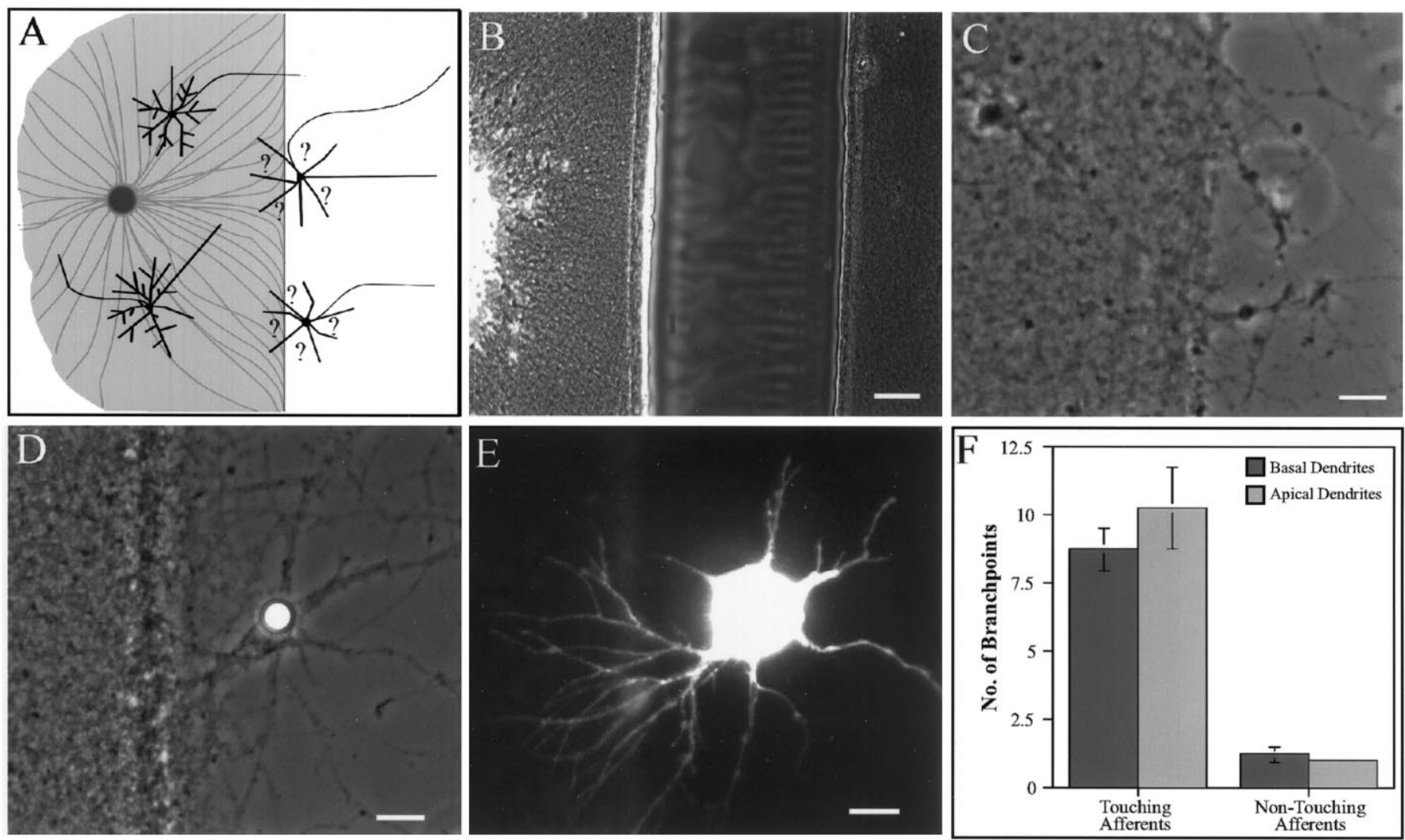

Figure 5. Localized induction of branching by afferents. $A$, Schematic illustration of our culture system, where neurons were grown along a border of afferents with one part of their dendritic tree touching the afferent net while the other part was growing on polylysine. $B$, Micrograph illustrating how axons extending from the explants (right and left lanes) formed sharp borders along a stripe of a nongrowth-permissive substrate (middle lane). $C$, Micrograph illustrating the presence of the afferent border even after removal of the nonadhesive substrate (right side). D, E, Example of a DiI-labeled neuron with phase contrast growing along a border of afferent axons for $11 \mathrm{~d}$, after the nonadhesive substrate had been peeled off. Only dendrites in contact with the afferents developed a rich branching pattern, as can be seen in the fluorescence picture. Dendrites from the same neuron, but growing on polylysine, remain unbranched. $F$, Quantification of branching in border neurons. Basal dendrites of all cell types $(n=32)$ as well as apical dendrites of pyramidal neurons $(n=11)$ developed branches only on those dendrites in contact with the afferents. This suggests that the afferents promote branching by means of a local effect on the dendrites rather than on the cell globally. Error bars represent SEM. Scale bars: $B, 500 \mu \mathrm{m} ; C-E, 20 \mu \mathrm{m}$.

cantly reduced from 13 spines per $100 \mu \mathrm{m}$ to 8 spines, $57 \%$ of the control group $(p<0.001)$ (Fig. 7). This significant reduction was observed in three of four sets of experiments, suggesting that all three glutamate receptors may be involved in the induction of spines.

\section{DISCUSSION}

Both branches and spines are important parameters for the structure and function of neurons. A high resolution cell culture system enabled us to investigate the role of afferents in development and differentiation of dendritic architecture in hippocampal neurons. We developed a well defined culture system providing a reliably high density of presynaptic innervation to individual neurons and eliminating the variability in innervation present in regular, dissociated cell cultures. Because formation of branches and spines are two relatively late events in the development of a dendritic tree, we used long-term cultures to avoid previous limitations posed by short-term cultures (Mattson et al., 1988; Cabell and Audesirk, 1993). We have found that the presence of afferents is indeed a necessary condition for the formation of dendritic branches and spines. Afferent innervation can induce branching via a non-activity-dependent mechanism. In contrast, mechanisms underlying the formation of dendritic spines seem to be activity-dependent and are most likely glutamate receptor-dependent.

\section{Branching}

Our study shows clearly that afferents can induce the formation of dendritic branches in hippocampal neurons, apparently independent of neuronal activity or the activation of glutamate receptors. Compared with single, non-innervated, or sparsely innervated cells, high levels of innervation lead to a fourfold increase in dendritic branching, with up to 40 dendritic branch points per neuron. The presence of TTX, a blocker of spontaneous neuronal activity, and the simultaneous presence of three major glutamate receptor blockers did not affect the increase of dendritic branching induced by axonal afferents, demonstrating that neither neuronal activity nor activation of glutamate receptors is necessary for the induction of branches. Our results, however, do not rule out a general involvement of neuronal activity and glutamate in affecting dendritic branching, as has been suggested previously (Smith and Jahr, 1992). In preliminary patch recordings performed on hippocampal neurons growing on the axonal net, little EPSC activity was recorded (data not shown). This indicates that levels of activity might have been too low to effectively influence dendritic branching, which might explain the ineffectiveness of 

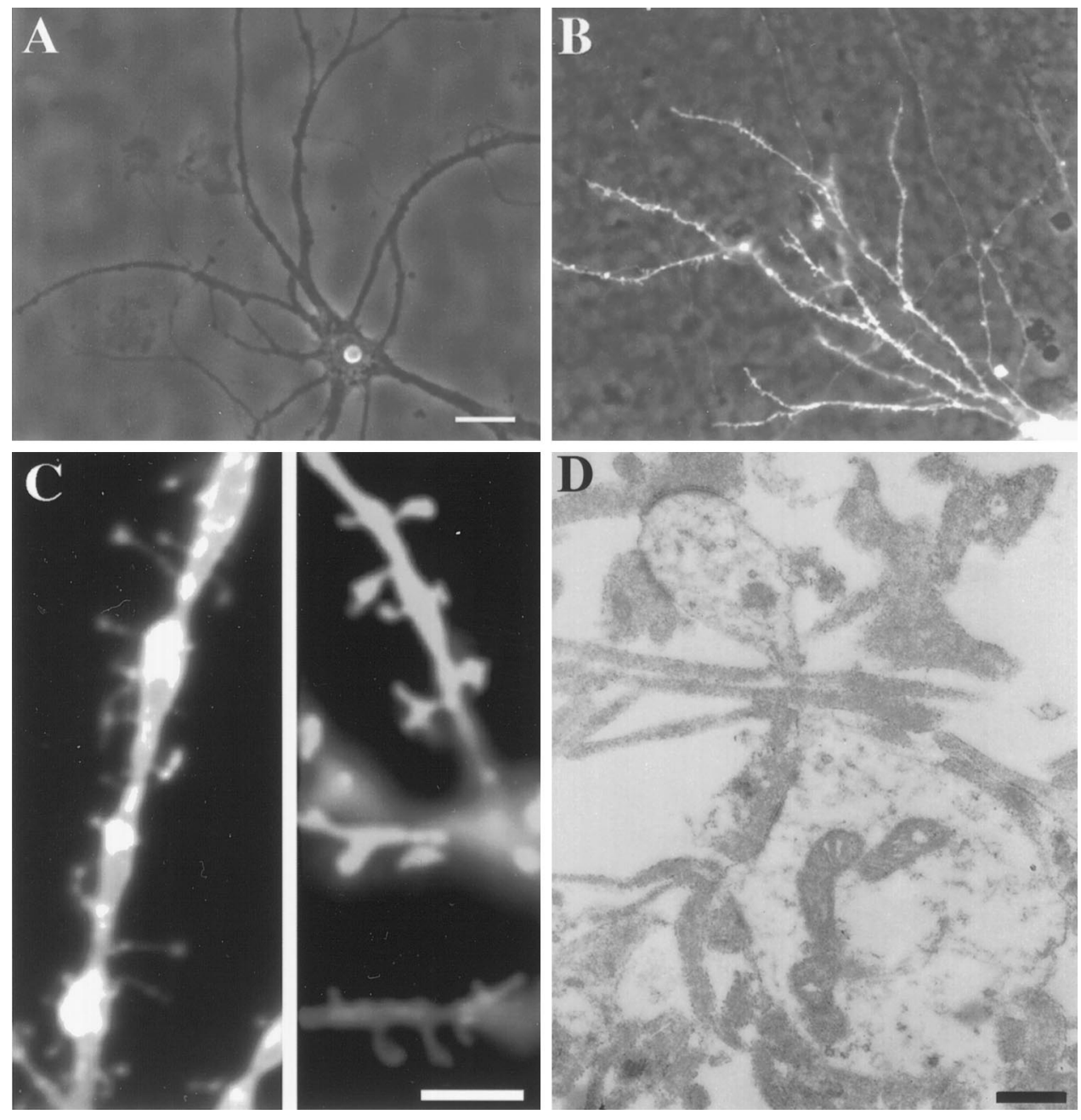

Figure 6. Development of dendritic spines in the presence of innervation. Micrograph of hippocampal neurons growing off $(A)$ and on $(B)$ the entorhinal axonal net. Only neurons growing in contact with the entorhinal axons develop numerous spines. $C$, Fluorescent micrograph showing spines of DiI-stained neurons at higher magnification. $D$, Electron micrograph of a spine. Scale bars: $A, B, 25 \mu \mathrm{m} ; C, 5 \mu \mathrm{m} ; D, 0.5 \mu \mathrm{m}$.

the blocker experiments. More importantly, however, our results strongly suggest that afferents provide as yet unidentified signals in addition to neuronal activity. Potential signals could include release of trophic substances across synapses, the expression of specific molecules associated with the formation of synapses, or the expression of specific surface molecules along axons.

Vaughn (1989) has proposed a synaptotrophic hypothesis which suggests that the formation of synapses may have subse-

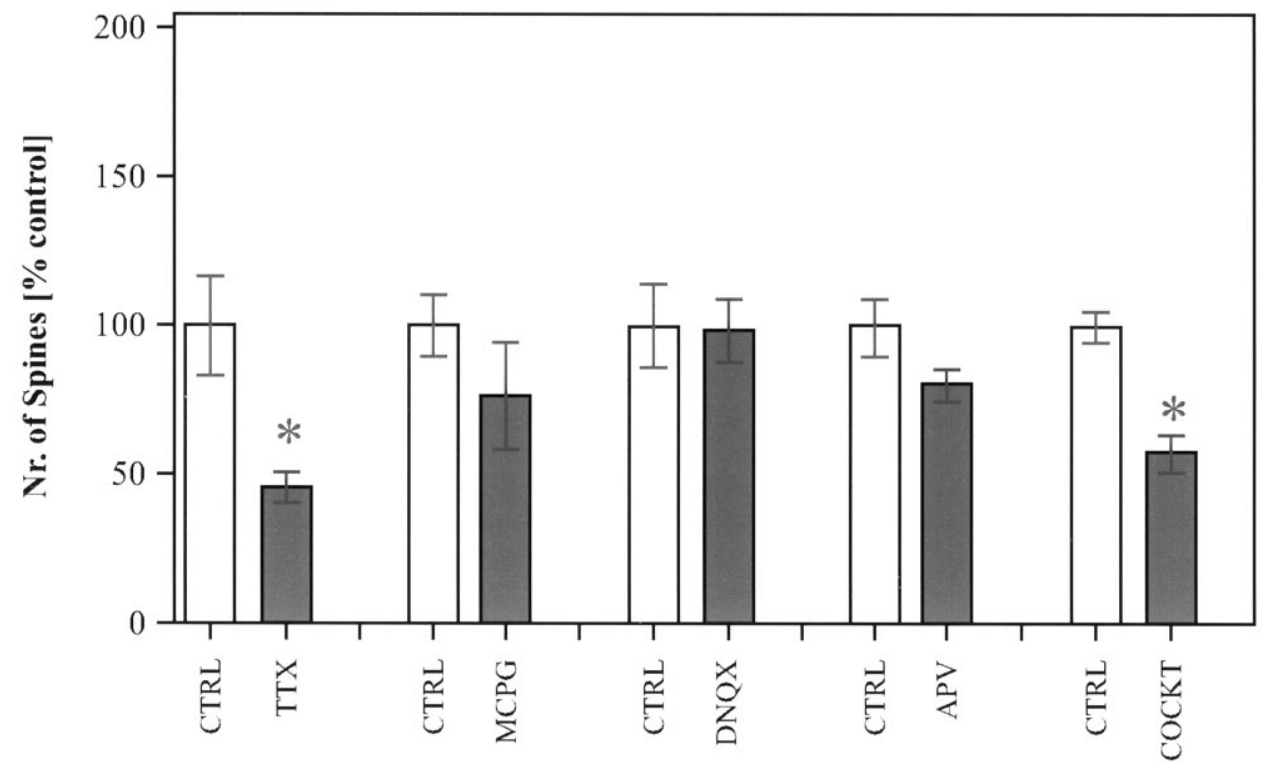

Figure 7. Role of activity and glutamate receptors in the development of dendritic spines. Chronic blockade of spontaneous activity by TTX $(n=20)$ significantly reduced the number of spines that developed after $14 \mathrm{~d}$ in vitro. Chronic blockade of individual glutamate receptors with different inhibitors $(D N Q X, n=26 ; A P V, n=23$; $M C P G, n=21)$ alone did not significantly decrease the number of spines; however, combination of all three blockers (COCKT, $n=80$ ) again significantly reduced the number of spines, suggesting a conjoint action of different glutamate receptors in the induction of spines. The average number of spines in treated cultures is shown as percentage of control. (Average number of spines in controls per $100 \mu \mathrm{m}$ : $12.6 \pm 0.8$; $n=101$.) Error bars represent SEM. 
quent effects on dendritic outgrowth and differentiation such as dendritic branching. Our results indicate that the emergence of dendritic branches may indeed be linked to the formation of synapses. First, induction of branches was specific for contact with afferents and did not occur with other cells such as astrocytes. Second, synaptophysin staining showed that synapses were formed between hippocampal neurons and the axonal net. Third, hippocampal neurons growing on a fixed carpet of axons did not develop branches. Thus, our results indicate that the signal necessary for branching is provided only by living axons and may therefore be closely associated with the formation of synapses between the entorhinal axons and hippocampal neurons. Although many different signals could mediate such a synaptotrophic effect, there is increasing evidence for neurotrophins as candidates. Recently it has been shown that the release of neurotrophins may be associated with the location of the synapse (Thoenen, 1995; von Bartheld et al., 1996). Furthermore, recent studies demonstrated that neurotrophins such as NT3, BDNF, and NT4 can influence the architecture of cortical neurons, including an increase in the number of dendrites and the formation of dendritic branches (McAllister et al., 1995). Hippocampal neurons have also been shown to express receptors for different neurotrophins and to change the expression of certain markers, such as calbindin, after application of neurotrophins (Ip et al., 1993). Although neurotrophins and other growth factors (Withers et al., 1995) clearly play a role in dendritic differentiation, their cellular source has not yet been identified; however, it is feasible that axonal afferents shape the dendritic architecture of hippocampal neurons by releasing trophic factors. Our experiments do not completely rule out that axons may provide an important adhesive substrate for dendrites, which may induce the formation of dendritic branches. Fixed axons did not promote dendritic branching, suggesting that axon-specific surface molecules may not be involved. Such potential surface molecules, however, may have lost their inductive capacity on fixation, or their expression may be associated specifically with the formation of synapses. Future experiments will therefore attempt to identify potential signals involved in dendritic branching associated with axonal afferents.

Whatever signal the afferents may provide to induce dendritic branching, our results show that this signal must act locally on the dendrites. By growing dendrites of the same individual hippocampal neurons on two different substrates, axonal afferents or polylysine, we demonstrated that branches formed only on dendrites interacting with afferents. This local action is similar to observations made in the auditory system in vivo. Here, sets of afferents are arranged in such a way that they innervate only parts of the dendritic tree of neurons in the nucleus laminaris. Removal of one set of afferents influenced only denervated dendrites to become atrophic and left other dendrites of the same cell intact (Gray et al., 1982; Smith et al., 1983). While this shows a local action of afferents in maintaining postsynaptic structures, our results demonstrate the importance of afferents during differentiation. Afferents exert their influence on the differentiation of the dendritic tree by a localized action rather than by a global effect on the overall maturation of the entire cell. This localized effect strongly suggests a specific signal, because it allows presynaptic afferents to select the site of postsynaptic differentiation. Because dendritic spines are intimately connected with synapses, we suspected that afferent innervation played a role in local spine formation as well. Preliminary experiments suggested that induction of spines was restricted to innervated dendrites within a neuron, but stripe experiments did not provide sharp enough borders for a long enough period of time (high density of spines developed only after $11 \mathrm{~d}$ ).

Although afferents seem to be a source of major extrinsic cues for the development of their postsynaptic target neurons, an intrinsic program cannot be excluded. This program very likely sets certain limits for the action of extrinsic influences and ultimately determines what cell type and hence what overall structure a cell will develop. Nonetheless, afferents seem to influence dendritic architecture by providing specific signals that enable dendrites to establish appropriate connections with their surrounding environment.

\section{Spines}

While formation of dendritic branches was not affected by blockade of spontaneous activity with TTX or a cocktail of glutamate receptor blockers, development of spines was altered. Significantly fewer spines developed in the presence of TTX. This result is in agreement with previous studies performed in various different culture systems (Annis et al., 1994; Wu et al., 1994). The presumably low level of activity in our cultures (mentioned above) together with the close association of spines with the postsynaptic site suggests that spines, in contrast to branches, may be sensitive to and respond to even small changes in neuronal activity. These could result in changes in the amount of neurotransmitter released, alterations in the release of trophic substances, or the altered expression of synaptic molecules.

Because spines emerge at the sites of synaptic contact, activity may play a role in spine formation through activation of postsynaptic glutamate receptors. We therefore tested the effects of three kinds of glutamate receptor blockers on the development of spines. All of these glutamate receptors are known to affect and modulate synaptic transmission. DNQX and AP-5 are specific blockers for the respective AMPA and NMDA glutamate receptors. MCPG is a broadly acting blocker for different types of metabotropic glutamate receptors. AMPA as well as NMDA receptors are located on the postsynaptic site of synapses (Benke et al., 1993; Craig et al., 1993), and they mediate postsynaptic transmission in the hippocampus (Bekkers and Stevens, 1989). Metabotropic glutamate receptors are present on the postsynaptic membrane, and their activation or blockade can alter calcium levels within hippocampal neurons (Baude et al., 1993; Frenguelli et al., 1993). Surprisingly, all three types of glutamate receptor blockers by themselves caused only a small but insignificant reduction in the formation of spines. Only the combination of all three blockers resulted in a significant reduction in the number of newly formed spines, which was comparable to the effect seen with the TTX treatment.

A general unspecific or cumulative toxic effect of all three blockers seems to be unlikely, because neither growth of the neurons nor dendritic branching was altered in the presence of the cocktail. It is rather intriguing to assume that glutamate receptors use a common mechanism to induce spine formation. Therefore only complete blockade of this mechanism would result in significant reduction of spines, which could explain why application of a glutamate blocker cocktail is needed to reduce the number of developing spines. All three glutamate receptor types tested in this study are able to activate calcium entry into dendrites. Activation of AMPA receptors can cause calcium influx via activation of voltage-sensitive calcium channels present on dendrites (Westenbroek et al., 1990; Mills et al., 1994). Activation of NMDA receptors can increase intracellular calcium 
levels through influx through the receptor-channel complex (Regehr et al., 1989). Finally, group 1 metabotropic glutamate receptors present on the postsynaptic site of hippocampal neurons can activate the release of calcium from intracellular stores (Martin et al., 1992; Baude et al., 1993; Frenguelli et al., 1993). It has been demonstrated that both synaptic input and glutamate are able to cause local increases in calcium in mature spines and might induce local changes in the structure and function of spines (Guthrie et al., 1991; Muller and Connor, 1991; Segal, 1996). So far the significance of glutamate and calcium for the formation and development of new spines has been unclear. Our results suggest that glutamate receptors may be involved in the induction of new spines. Because each individual glutamate receptor is able to cause increases in calcium, only the combined blockade of two or more receptors may be sufficient to eliminate local calcium increases at the postsynaptic site, ultimately coupling activity to cytoskeletal changes necessary for spine formation.

There may be another explanation for a combined effect of the glutamate receptor blockers; that is, the antagonists were actually acting on the explant, upstream of the actual synapses we were studying. By blocking synaptic interactions between neurons within the entorhinal cortical explant, the antagonists may reduce afferent activity in hippocampal neurons in a way similar to that caused by TTX. How could afferent activity exert its effect on the formation of spines independent of the activation of postsynaptic glutamate receptors? Such an alternative mechanism could either work through activity-dependent release of trophic molecules or activity-dependent expression of molecules important for differentiation of the synaptic site. Surface molecules are important for early differentiation and alignment of pre- and postsynaptic sites in muscle (Nastuk and Fallon, 1993; Goodearl et al., 1995). Their significance in the formation of synapses within the CNS is completely speculative so far, although molecules have been found that are specifically associated with synaptic membranes in the brain (Kennedy et al., 1983; Lasher et al., 1988; Ushkaryov et al., 1992; Ichtchenko et al., 1995). Such molecules may be important not only for early alignment of pre- and postsynaptic sites but also for later events in the differentiation of the synapse, such as spine formation. Activity-dependent changes in the expression of these molecules could potentially link presynaptic activity to the induction of postsynaptic events, such as the formation of spines without the involvement of transmitter release or any involvement of postsynaptic glutamate receptors. The presence of such additional regulatory mechanisms is supported by the fact that activity as well as glutamate receptor blockade did not completely abolish the formation of spines in our experiments but only reduced it by $\sim 50 \%$. Ultimately the role of glutamate receptors can only be determined by a preparation that separates pre- and postsynaptic sites in a way that allows them to be targeted individually.

In conclusion, our results demonstrate the significance of afferents in the development of dendritic architecture. They support the notion that there is a tight interplay between presynaptic innervation and postsynaptic differentiation. Our experiments show that afferents are able to induce branching and spines locally on individual dendrites. Furthermore, activity and the activation of glutamate receptors was necessary only for the formation of spines and not for branching. This strongly suggests the presence of multiple signals emerging from presynaptic afferents that are involved in the differentiation of dendrites and ultimately determine dendritic architecture.

\section{REFERENCES}

Annis CA, Dowd DKO, RT Robertson (1994) Activity-dependent regulation of dendritic spine density on cortical pyramidal neurons in organotypic slice cultures. J Neurobiol 25:1483-1493.

Anderson WA, Flumerfelt BA (1984) Long-term effect of mossy fiber degeneration in the rat. J Comp Neurol 227:414-423.

Banker GA, Cowan WM (1979) Further observations on hippocampal neurons in dispersed cell culture. J Comp Neurol 187:469-494.

Baptista CA, Hatten ME, Blazeski R, Mason CA (1994) Cell-cell interactions influence survival and differentiation of purified Purkinje cells in vitro. Neuron 12:243-260.

Baude A, Nusser Z, Roberts JD, Mulvihill E, McIlhinney RA, Somogyi P (1993) The metabotropic glutamate receptor (mGluR1 alpha) is concentrated at perisynaptic membrane of neuronal subpopulations as detected by immunogold reaction. Neuron 11:771-787.

Bekkers JM, Stevens CF (1989) NMDA and non-NMDA receptors are co-localized at individual excitatory synapses in cultured rat hippocampus. Nature 341:230-233.

Benke TA, Jones OT, Collingridge GL, Angelides KJ (1993) $N$-methylD-aspartate receptors are clustered and immobilized on dendrites of living cortical neurons. Proc Natl Acad Sci USA 90:7819-7823.

Benson DL, Watkins FH, Steward O, Banker G (1994) Characterization of GABAergic neurons in hippocampal cell cultures. J Neurocytol 23:279-295.

Cabell L, Audesirk G (1993) Effects of selective inhibition of protein kinase $\mathrm{C}$, cyclic AMP-dependent protein kinase, and $\mathrm{Ca}(2+)$ calmodulin-dependent protein kinase on neurite development in cultured rat hippocampal neurons. Int J Dev Neurosci 11:357-368.

Craig AM, Blackstone CD, Huganir RL, Banker G (1993) The distribution of glutamate receptors in cultured rat hippocampal neurons: postsynaptic clustering of AMPA-selective subunits. Neuron 10:1055-1068.

Dailey ME, Smith SJ (1996) The dynamics of dendritic structure in developing hippocampal slices. J Neurosci 16:2983-2994.

Dan Y, Lo Y, Poo MM (1995) Plasticity of developing neuromuscular synapses. Prog Brain Res: 105:211-215.

Deitch JS, Rubel EW (1984) Afferent influences on brain stem auditory nuclei of the chicken: time course and specificity of dendritic atrophy following deafferentation. J Comp Neurol 229:66-79.

Frenguelli BG, Poteir B, Slater NT, Alford S, Collingridge GL (1993) Metabotropic glutamate receptors and calcium signalling in dendrites of hippocampal CA1 neurons. Neuropharmacology 32:1229-1237.

Frotscher M (1983) Dendritic plasticity in response to partial deafferentation. In: Neurobiology of the hippocampus (Seifert W, ed), pp 65-80. New York: Academic.

Frotscher M, Hamori J, Wenzel J (1977) Transneuronal effects of entorhinal lesions in the early postnatal period on synaptogenesis in the hippocampus of the rat. Exp Brain Res 30:549-560.

Frotscher M, Nitsch C, Hassler R (1981) Synaptic reorganization n the rabbit hippocampus after lesion of commissural afferents. Anat Embryol 163:15-30.

Goodearl AD, Yee AG, Sandrock Jr AW, Corfas G, Fischbach GD (1995) ARIA is concentrated in the synaptic basal lamina of the developing chick neuromuscular junction. J Cell Biol 130:1423-1434.

Gray L, Smith Z, Rubel EW (1982) Developmental and experimental changes in dendritic symmetry in n. laminaris of the chick. Brain Res 244:360-364.

Guthrie PB, Segal M, Kater SB (1991) Independent regulation of calcium revealed by imaging dendritic spines. Nature 354:76-80.

Hamori J (1973) The inductive role of presynaptic axons in the development of postsynaptic spines. Brain Res 62:337-344.

Harris KM, Kater SB (1994) Dendritic spines: cellular specializations imparting both stability and flexibility to synaptic function. Annu Rev Neurosci 17:341-371.

Horner CH (1993) Plasticity of the dendritic spine. Prog Neurobiol 41:281-321.

Ichtchenko K, Hata Y, Nguyen T, Ullrich B, Missler M, Moomaw C, Sudhof TC (1995) Neuroligin 1: a splice site-specific ligand for betaneurexins. Cell 81:435-443.

Ip NY, Li Y, Yancopoulos GD, Lindsay RM (1993) Cultured hippocampal neurons show responses to BDNF, NT-3, and NT-4, but not NGF. J Neurosci 13:3394-3405.

Katz LC, Constantine-Paton M (1988) Relationships between segregated afferents and postsynaptic neurons in the optic tectum of threeeyed frogs. J Neurosci 8:3160-3180. 
Katz LC, Gilbert CD, Wiesel TN (1989) Local circuits and ocular dominance columns in monkey striate cortex. J Neurosci 9:1389-1399.

Kennedy MB, Bennett MK, Erondu NE (1983) Biochemical and immunochemical evidence that the "major postsynaptic density protein" is a subunit of a calmodulin-dependent protein kinase. Proc Natl Acad Sci USA 80:7357-7361.

Koch C, Zador A (1993) The function of dendritic spines: devices subserving biochemical rather than electrical compartmentalization. J Neurosci 13:413-422.

Kossel A, Löwel S, Bolz J (1995) Relationships between dendritic fields and functional architecture in striate cortex of normal and visually deprived cats. J Neurosci 15:3913-3926.

Lasher RS, Erickson PF, Mena EE, Cotman CW (1988) The binding of a monoclonal antibody reactive with pp60(v-src) to the rat CNS both in vitro and in vivo: evidence that the epitope is present intracellularly as well as being associated with a number of antigenically related polypeptides located externally in the plasma membrane only in the synaptic region. Brain Res 452:184-202.

Martin LJ, Blackstone CD, Huganir RL, Price DL (1992) Cellular localization of a metabotropic glutamate receptor in rat brain. Neuron 9:259-270.

Mattson MP, Lee RE, Adams ME, Guthrie PB, Kater SB (1988) Interactions between entorhinal axons and target hippocampal neurons: a role for glutamate in the development of hippocampal circuitry. Neuron 1:865-876.

McAllister AK, Lo DC, Katz LC (1995) Neurotrophins regulate dendritic growth in developing visual cortex. Neuron 15:791-803.

Miller JP, Rall W, Rinzel J (1985) Synaptic amplification by active membrane in dendritic spines. Brain Res 325:325-330.

Mills LR, Niesen CE, So AP, Carlen PL, Spigelman I, Jones OT (1994) N-type $\mathrm{Ca}^{2+}$ channels are located on somata, dendrites, and a subpopulation of dendritic spines on live hippocampal pyramidal neurons. J Neurosci 14:6815-6824.

Muller W, Connor JA (1991) Dendritic spines as individual neuronal compartments for synaptic Ca2+ responses. Nature 354:73-76.

Nastuk MA, Fallon JR (1993) Agrin and the molecular choreography of synapse formation. Trends Neurosci 16:72-76.

Papa M, Segal M (1996) Morphological plasticity in dendritic spines of cultured hippocampal neurons. Neuroscience 71:1005-1011.

Parnavelas JG, Lynch G, Brecha N, Cotman CW, Globus A (1974) Spine loss and regrowth in hippocampus following deafferentation. Nature 248:71-73.

Regehr WG, Connor JA, Tank DW (1989) Optical imaging of calcium accumulation in hippocampal pyramidal cells during synaptic activation. Nature 341:533-536.

Segal M (1996) Imaging of calcium variations in living dendritic spines of cultured rat hippocampal neurons. J Physiol (Lond) 486:283-295.

Shatz CJ (1990) Impulse activity and the patterning of connections during CNS development. Neuron 5:745-756.

Smith SJ, Jahr CE (1992) Rapid induction of filopodial sprouting by application of glutamate to hippocampal neurons. In: The nerve growth cone (Letourneau PC, Kater SB, Macagno ER, eds), pp 19-26. New York: Raven.

Smith ZDJ, Gray L, Rubel EW (1983) Afferent influences on brainstem auditory nuclei in the chicken: n. laminaris dendritic length following monaural conductive hearing loss. J Comp Neurol 220:199-205.

Spruston N, Schiller Y, Stuart G, Sakmann B (1995) Activity-dependent action potential invasion and calcium influx into hippocampal CA1 dendrites. Science 268:297-300.

Thoenen H (1995) Neurotrophins and neuronal plasticity. Science 270:593-598.

Ushkaryov YA, Petrenko AG, Geppert M, Sudhof TC (1992) Neurexins: synaptic cell surface proteins related to the alpha-latrotoxin receptor and laminin. Science 257:50-56.

Vaughn JE (1989) Review: fine structure of synaptogenesis in the vertebrate central nervous system. Synapse 3:255-285.

von Bartheld CS, Byers MR, Williams R, Bothwell M (1996) Anterograde transport of neurotrophins and axodendritic transfer in the developing visual system. Nature 379:830-833.

Westenbroek RE, Ahlijanian MK, Catterall WA (1990) Clustering of L-type $\mathrm{Ca} 2+$ channels at the base of major dendrites in hippocampal pyramidal neurons. Nature 347:281-284.

Withers G, Higgins D, Rueger D, Banker G (1995) Osteogenic protein-1 (OP-1) induces dendritic growth and branching in cultured hippocampal neurons. Soc Neurosci Abstr 21:1542.

Wu T, Maguire MJ, Ebner FF (1994) Blockade of spontaneous neural activity impairs the development of the dendritic spines in culture. Soc Neurosci Abstr 20:1298.

Yuste R, Denk W (1995) Dendritic spines as basic functional units of neuronal integration. Nature 375:682-684.

Zafirov S, Heimrich B, Frotscher M (1994) Dendritic development of dentate granule cells in the absence of their specific extrinsic afferents. J Comp Neurol 345:472-480.

Ziv NE, Smith SJ (1996) Evidence for a role of dendritic filopodia in synaptogenesis and spine formation. Neuron 17:91-102. 